\title{
Evolutionary History and Diversity of Unionoid Mussels (Mollusca: Bivalvia) in the Japanese Archipelago
}

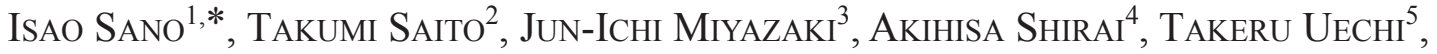 \\ TAKAKI KONDO ${ }^{6} \&$ SATOSHI CHIBA ${ }^{1,7}$ \\ ${ }^{1}$ Graduate School of Life Sciences, Tohoku University, 2-1-1 Katahira, Aoba, Sendai, Miyagi 980-8577, Japan \\ ${ }^{2}$ Department of Biology, Faculty of Science, Toho University, 2-2-1 Miyama, Funabashi, Chiba 274-8510, Japan \\ ${ }^{3}$ Faculty of Education, University of Yamanashi, 4-4-37 Takeda, Kofu, Yamanashi 400-8510, Japan \\ ${ }^{4}$ Musashi High School and Junior High School, 1-26-1 Toyotamakami, Nerima, Tokyo 176-8534, Japan \\ ${ }^{5}$ Faculty of Agriculture, Kindai University, 3327-204 Nakamachi, Nara, Nara 631-8505, Japan \\ ${ }^{6}$ Osaka Kyoiku University, 4-698-1 Asahigaoka, Kashiwara, Osaka 582-8582, Japan \\ ${ }^{7}$ Center for Northeast Asian Studies, Tohoku University, 41 Kawauchi, Aoba, Sendai, Miyagi 980-8576, Japan
}

Received 31 July 2019; Accepted 20 January 2020 Responsible Editor: Shigeaki Kojima

doi: $10.3800 /$ pbr. 15.97

\begin{abstract}
The evolutionary history and diversity of unionoid mussels in East Asia need to be clarified and would shed light on the formation process of the unique fauna of Japan. Unionoid mussels (Mollusca: Bivalvia) are unique models for understanding the process by which organisms have diversified before and after the formation of the Japanese archipelago. Unionoid mussels have poor dispersal ability, so it is thought that they would have been strongly influenced by the archipelago's formation. Therefore, the speciation and diversification processes of mussels before and after the archipelago's formation were investigated by analyzing the nuclear and mitochondrial DNA of a wide range of species, particularly those inhabiting East Asia. The evolutionary history and divergence time of these mussels were examined. Unionoid mussels were found to have higher endemicity than other freshwater organisms. Although most of the endemic unionoid mussels of Japan are likely to have diverged before the formation of the Japanese archipelago, some other Japanese unionoid mussel species, including species endemic to Lake Biwa, an ancient lake in Japan, potentially diverged after the Japanese archipelago began to separate from the continent. This suggest that adaptation to the unique habitat of the ancient lake has caused diversification in the mussels endemic to it.
\end{abstract}

Key words: divergence time, Margaritiferidae, nuclear DNA, phylogeny, Unionidae

\section{Introduction}

Located at the eastern end of the Eurasian continent, the Japanese archipelago is a biodiversity hotspot (Ceballos \& Brown 1995; Kerswell 2006; Fonseca 2009). The high biodiversity of the Japanese archipelago is seemingly due to the complex formation history of the Japanese fauna (Motokawa \& Kajihara 2016). Japanese freshwater fauna also have a complex formation history (Watanabe et al. 2006; Tojo et al. 2017). 1) The formation of the Japanese archipelago dates back to about 15 million years ago (Hamano \& Tosha 1985; Torii et al. 1985; Jolivet 1992). 2) Af-

\footnotetext{
*Corresponding author: Isao Sano; E-mail, sano75025@gmail.com
}

ter the formation of the archipelago, the freshwater fauna appeared to have interacted with continental fauna several times owing to the glacial-interglacial cycle (Cronin et al. 1994). 3) The Japanese archipelago has been historically affected by various climatic and environmental conditions because it is located in a wide latitude range spanning more than $3,000 \mathrm{~km}$ from north to south and has climate zones ranging from subarctic to subtropical (Kubota et al. 2017; Tojo et al. 2017). 4) Some regions and taxa in the archipelago are thought to have gone through large-scale extinctions (Nakajima 1986; Nakajima 1987; Watanabe \& Uyeno 1999).

In contrast, the histories of the geographical formation and the regional fauna of each taxon are not always consistent. It is important to understand whether the formation of 
freshwater fauna has been influenced mainly by common factors such as geographic isolations, or whether the dispersal capacity, interspecific relationships, and randomness of individual taxa have shaped freshwater fauna beyond such commonalities (Arbogast \& Kenagy 2001).

To clarify this, it is important to construct and compare the molecular phylogenies of various taxa collected from both the Asian continent and the Japanese archipelago. However, reports of comparative studies that consider the historical relationship between organisms living on the Asian continent and those living in the Japanese archipelago have not been fully accumulated. Many studies using several freshwater organisms (Insect: Suzuki et al. 2014; Saito et al. 2016, 2018. Fish: Miyazaki et al. 2011; Gao et al. 2012; Tominaga et al. 2016; Tsao et al. 2016; Kano et al. 2018. Mollusca: Park \& Kim 2003; Yamada et al. 2010; Hirano et al. 2015, 2019; Saito et al. 2018a, 2018c) have been conducted to verify the diversity of fauna in East Asia, mainly that of fauna in the Japanese archipelago. However, the evolutionary relationship between the fauna of the Asian continent and the Japanese archipelago has not been substantially clarified, with most studies having mainly used samples from the Japanese archipelago and only a few samples from the Asian continent. In recent years, studies using cyprinid fishes and planorbid snails have examined the process of faunal diversification using sufficient continental samples as well as samples from the Japanese archipelago (Saito et al. 2018b; Jang-Liaw et al. 2019). Small freshwater snails, such as planorbids, are passive dispersers that disperse across water systems via birds and within water systems via fishes (Rees 1965; Boag 1986; Wesselingh et al. 1999; Green \& Figuerola 2005; Kappes \& Haase 2012; van Leeuwen \& van der Velde 2012; van Leeuwen et al. 2012), whereas fishes are active dispersers that do not move outside water systems. Unionoid mussels are also passive dispersers that migrate within water systems; their larvae can disperse by attaching to fishes, though they do not move after larval settlement to the bottom of a water body. Levels of gene flow among populations of unionoid mussels are likely to reflect their passive dispersal ability. In addition, bitterling fishes have a unique trait of laying eggs in the gills of unionoid mussels, whereas unionoid larvae need to parasitize mainly freshwater fishes other than bitterlings (Smith et al. 2000a, 2000b, 2001; Mills \& Reynolds 2002a, 2002b; Itoh et al. 2003; Kitamura 2005, 2006a, 2006b; Mills et al. 2005; Reichard et al. 2006, 2007, 2010). Thus, unionoid mussels, unlike cyprinid fishes and planorbid snails, form close symbiotic relationships with other freshwater organisms and are central to biological interactions in freshwater. The evolutionary history of these mussels, therefore, appears to reflect more strongly the history of geographic formation than that of other freshwater organisms and is therefore a good model for examining the relationship between the formations of fauna and geography.

There are many freshwater-mussel species that have not been analyzed or have been studied only piecemeal, and there is an increasing need for more accurate research on individual taxa. Kano et al. (2019) reported a phylogenetic tree of specimens in Japan based on mitochondrial 16S rDNA, but the positions of almost all clades on the tree, except those of species-level clades, were poorly supported. Furthermore, the samples they used were limited to those from Kyushu and the Ryukyu Islands, making it difficult to understand the diversification process of unionoid mussels in the Japanese archipelago.

In contrast, Sano et al. (2017) constructed phylogenetic trees using species living across the Japanese archipelago and evaluated the current systematics of Japanese unionoid mussels. However, the evolutionary relationships between mussels living in continental East Asia and those living in the Japanese archipelago remain unclear, as do the changes in unionoid divergence before and after the archipelago's formation. Therefore, it is necessary to analyze nuclear and mitochondrial DNA using a comprehensive set of species inhabiting the Asian continent and the archipelago.

In this study, we estimated phylogenetic relationships based on nuclear and mitochondrial sequences. In addition, we estimated the divergence times of East Asian unionoid mussel species to elucidate how unionoid mussels diversified before and after the formation of the Japanese archipelago.

\section{Materials and Methods}

\section{Materials}

Seventy-three unionoid specimens were collected in Japan and preserved in $99.5 \%$ ethanol. Prior to our genetic study, we identified specimens based on the morphological features of the shell and larvae described by Kondo (2008, 2015) and Lopes-Lima et al. (2017) and assigned them to Margaritiferidae (2 species) and Unionidae (16 species). In addition, we selected specimens from each species belonging to the same families as those living in the Japanese archipelago and obtained their data from the GenBank database. Detailed information on the specimens and sample abbreviations, including those of samples used by Sano et al. (2017), are given in Table 1 .

\section{DNA sequencing}

To prepare samples for sequencing, we removed the foot muscle from each unionoid mussel and boiled it at $100^{\circ} \mathrm{C}$. Then, total DNA was extracted using a DNeasy ${ }^{\circledR}$ Blood \& Tissue Kit (QIAGEN GmbH, Hilden, Germany) according to the manufacturer's protocol. PCRs for nuclear $28 \mathrm{~S}$ rDNA using primers, 28S-RD1.3f and 28S-rD4b (Whiting 2002), were conducted under the following condition: initial denaturation at $95^{\circ} \mathrm{C}$ for $3 \mathrm{~min}$; 35 cycles of denaturation at $95^{\circ} \mathrm{C}$ for $45 \mathrm{sec}$, annealing at $60^{\circ} \mathrm{C}$ for $3 \mathrm{~min}$, and extension at $72^{\circ} \mathrm{C}$ for $30 \mathrm{sec}$; followed by a final extension at $72^{\circ} \mathrm{C}$ for $7 \mathrm{~min}$. Mitochondrial COI DNA was amplified 
Table 1. Specimens used in phylogenetic analyses (*: data obtained from GenBank).

\begin{tabular}{|c|c|c|c|c|c|c|c|}
\hline \# & Family & Species & Sample Ab. & Locality & $\begin{array}{l}\text { Accession } \\
\text { No. of } \\
\text { mitochondrial } \\
\text { COI DNA }\end{array}$ & $\begin{array}{l}\text { Accession } \\
\text { No. of } \\
\text { mitochondrial } \\
\text { 16S rDNA }\end{array}$ & $\begin{array}{l}\text { Accession } \\
\text { No. of } \\
\text { nuclear } \\
\text { 28S rDNA }\end{array}$ \\
\hline U1 & Margaritiferidae & Margaritifera laevis & Ma14-1SUTO & Gujo, Gifu, Japan & LC518956 & LC223972* & \\
\hline $\mathrm{U} 2$ & & & Ma14-2SUTO & Gujo, Gifu, Japan & LC518957 & LC223973* & LC519050 \\
\hline U3 & & & Ma14-3SUTO & Gujo, Gifu, Japan & LC518958 & LC223974* & \\
\hline U4 & & & Ma-u2 & Teshiogawa River, Hokkaido, Japan & LC518959 & LC519029 & LC519051 \\
\hline U5 & & & - & Iwaizumi, Japan & KU763223* & EU590915* & \\
\hline U6 & & & - & Iwaizumi, Honshu, Japan & KU763221* & EU590914* & \\
\hline U7 & & & - & Iwaizumi, Town, Honshu, Japan & KU763222* & KU763192* & \\
\hline U8 & & Margaritifera togakushiensis & Mt32-04 & Togakushi, Nagano, Japan & LC518960 & LC519030 & LC519052 \\
\hline U9 & & & Mt-k & Togakushi, Nagano, Japan & LC518961 & LC224020* & LC519053 \\
\hline U10 & & & - & Togakushi, Nagano, Japan & KU763244* & KU763215* & \\
\hline U11 & & & - & Togakushi, Nagano, Japan & KU763245* & KU763216* & \\
\hline U12 & & Margaritifera marrianae & - & Hunter, Creek, Alabama, USA & KU763243* & KU763214* & \\
\hline U13 & & Margaritifera hembeli & - & Valentine, Creek, Louisiana, USA & KU763218* & KU763189* & \\
\hline U14 & & & - & Brown, Creek, Louisiana, USA & KU763219* & KU763190* & \\
\hline $\mathrm{U} 15$ & & Margaritifera dahurica & - & Komissarovka River, Primorye, Terr, Russia & AY579123* & KF514426* & \\
\hline U16 & & Margaritifera margaritifera & - & Carrabassett River, Maine, USA & KU763234* & KU763203* & \\
\hline U17 & & & - & Thurma River, Kola Peninsula, Russia & AF303334* & AF303296* & \\
\hline U18 & & & - & Locust Creek, Pennsylvania, USA & KU763227* & KU763196* & \\
\hline U19 & & & - & Regen River, Germany & KU763235* & KU763204* & \\
\hline $\mathrm{U} 20$ & & & - & Nore River, Ireland & AF303343* & AF $303302^{*}$ & \\
\hline $\mathrm{U} 21$ & & & - & Nore River, Ireland & AF $303342 *$ & AF303301* & \\
\hline $\mathrm{U} 22$ & & & - & Dereen River, Hacketstown, Ireland & KU763237* & AF303293* & \\
\hline $\mathrm{U} 23$ & & & - & Varzuga River, Kola Peninsula, Russia & KU763238* & KU763208* & \\
\hline $\mathrm{U} 24$ & & & - & Varzuga River, Kola Peninsula, Russia & KU763239* & KU763209* & \\
\hline $\mathrm{U} 25$ & & & - & Suomujoki, Finnland & KU763230* & KU763199* & \\
\hline $\mathrm{U} 26$ & & & - & Salmon Stream, Maine, USA & KU763232* & KU763201* & \\
\hline $\mathrm{U} 27$ & & & - & Salmon Stream, Maine, USA & KU763233* & KU763202* & \\
\hline $\mathrm{U} 28$ & & Margaritifera rochechouartii & - & Nanxinxiang, Gan River, Jiangxi, China & MF072499* & MF072506* & \\
\hline $\mathrm{U} 29$ & & & - & Nanxinxiang, Gan River, Jiangxi, China & MF072498* & MF072505* & \\
\hline $\mathrm{U} 30$ & & & - & Nanxinxiang, Gan River, Jiangxi, China & MF072500* & MF072507* & \\
\hline $\mathrm{U} 31$ & & & - & Nanxinxiang, Gan River, Jiangxi, China & MF072501* & MF072508* & \\
\hline $\mathrm{U} 32$ & & & - & Nanxinxiang, Gan River, Jiangxi, China & MF072502* & MF072509* & \\
\hline U33 & & & - & Nanxinxiang, Gan River, Jiangxi, China & & & MG595548* \\
\hline U34 & & Margaritifera laosensis & - & Mun River, Thailand & KU763224* & KU763193* & \\
\hline $\mathrm{U} 35$ & & & - & Luang Prabang, Laos & KU763225* & KU763194* & \\
\hline U36 & & Margaritifera auricularia & - & Canal Imperial Zaragoza, Spain & AF303309* & AF303274* & \\
\hline U37 & & & - & Ebro River, Tarragona, Spain & AY $579125^{*}$ & AY $579083^{*}$ & \\
\hline U38 & & & - & Canal Imperial Zaragoza, Spain & AF303313* & AF303278* & \\
\hline U39 & & Margaritifera marocana & - & Oum Er Rbia River, Dange Bradia, Morocco & EU429677* & EU429687* & \\
\hline U40 & & & - & Oum Er Rbia River, Dange Bradia, Morocco & EU429678* & EU429689* & \\
\hline U41 & & & - & Abid River, Imadahine Oued Abid, Morocco & EU429679* & EU429691* & \\
\hline U42 & & Margaritifera falcata & - & Idaho, USA & AY 579128* & AY579085* & \\
\hline U43 & & Cumberlandia monodonta & - & Missouri, USA & AY579131* & AY579089* & \\
\hline $\mathrm{U} 44$ & Unionidae & Scabies crispata & - & Southeast Asia & KP795023* & $\mathrm{KX} 713253^{*}$ & \\
\hline $\mathrm{U} 45$ & & Nodularia douglasiae & BIWATATE 3 & Lake Biwa, Shiga, Japan & LC518962 & LC223961* & LC519054 \\
\hline U46 & & & KAWATATE 2 & Lake Kawaguchiko, Yamanashi, Japan & LC518963 & LC223964* & \\
\hline U47 & & & KAWATATE 6 & Lake Kawaguchiko, Yamanashi, Japan & LC518964 & LC223965* & \\
\hline U48 & & & Un40-01 & Wakayama, Japan & LC518965 & LC223975* & \\
\hline U49 & & & Un40-02 & Wakayama, Japan & LC518966 & LC223976* & \\
\hline U50 & & & Un43-06f & Nakama, Fukuoka, Japan & LC518967 & LC223977* & LC519055 \\
\hline U51 & & & Un43-07f & Nakama, Fukuoka, Japan & LC518968 & LC223978* & \\
\hline U52 & & & Un-u5 & Yodo River, Osaka, Japan & LC518969 & LC519031 & \\
\hline U53 & & & Un-u6 & Mukogawa River, Hyogo, Japan & LC518970 & LC519032 & \\
\hline U54 & & & - & China & $\mathrm{NC} 026111^{*}$ & $\mathrm{NC} 026111^{*}$ & \\
\hline U55 & & & - & China & KM657954* & KM657954* & \\
\hline U56 & & & - & Gan River, Jiangxi, China & & & MG595555* \\
\hline U57 & & Unio delphinus & - & Portugal & NC033854* & NC033854* & \\
\hline U58 & & Unio pictorum & - & England, UK & $\mathrm{KC} 429109^{*}$ & KC429266* & \\
\hline U59 & & Unio crassus & - & Europe & NC033976* & NC033976* & \\
\hline
\end{tabular}


Table 1. Continued.

\begin{tabular}{|c|c|c|c|c|c|c|c|}
\hline \# & Family & Species & Sample Ab. & Locality & $\begin{array}{l}\text { Accession } \\
\text { No. of } \\
\text { mitochondrial } \\
\text { COI DNA }\end{array}$ & $\begin{array}{l}\text { Accession } \\
\text { No. of } \\
\text { mitochondrial } \\
\text { 16S rDNA }\end{array}$ & $\begin{array}{l}\text { Accession } \\
\text { No. of } \\
\text { nuclear } \\
\text { 28S rDNA }\end{array}$ \\
\hline U60 & & Cuneopsis pisciculus & - & China & NC026306* & NC026306* & \\
\hline U61 & & Cuneopsis celtiformis & - & Gan River, Jiangxi, China & & & MG595492* \\
\hline U62 & & Lamprotula gottschei & - & China & NC023806* & NC023806* & \\
\hline U63 & & Inversiunio reinianus & Ir07-01 & Lake Biwa, Shiga, Japan & LC518971 & LC223979* & \\
\hline U64 & & & Ir07-k10 & Lake Biwa, Shiga, Japan & LC518972 & LC519033 & LC519056 \\
\hline U65 & & & Ir07-k11 & Lake Biwa, Shiga, Japan & LC518973 & LC519034 & LC519057 \\
\hline U66 & & & Ir07-k12 & Lake Biwa, Shiga, Japan & LC518974 & LC519035 & \\
\hline U67 & & & Ir07-k13 & Lake Biwa, Shiga, Japan & LC518975 & LC519036 & \\
\hline U68 & & & Ir07-k14 & Lake Biwa, Shiga, Japan & LC518976 & LC519037 & \\
\hline U69 & & Inversiunio jokohamensis & Ij25-01 & Sakai, Yamagata, Japan & LC518977 & LC223980* & \\
\hline $\mathrm{U} 70$ & & & Ij25-09 & Sakai, Yamagata, Japan & LC518978 & LC223981* & LC519058 \\
\hline U71 & & & Ij21-28f & Lake Anenuma, Aomori, Japan & LC518979 & LC223982* & LC519059 \\
\hline U72 & & & Ij08-01 & Lake Kitaura, Ibaraki, Japan & LC518980 & LC223984* & \\
\hline U73 & & & NiiYoko1 & Niigata, Japan & LC518981 & LC519038 & \\
\hline U74 & & & NiiYoko2 & Niigata, Japan & LC518982 & LC519039 & \\
\hline U75 & & & NiiYoko3 & Niigata, Japan & LC518983 & LC519040 & \\
\hline U76 & & & NiiYoko4 & Niigata, Japan & LC518984 & LC519041 & \\
\hline U77 & & & NiiYoko6 & Niigata, Japan & LC518985 & LC519042 & \\
\hline U78 & & Inversiunio yanagawensis & Iy09-10 & Gion, Okayama, Japan & LC518986 & LC223987* & LC519060 \\
\hline U79 & & & Iy43-01 & Fukuoka, Japan & LC518987 & LC223988* & \\
\hline U80 & & & Iy43-05f & Fukuoka, Japan & LC518988 & LC223989* & LC519061 \\
\hline U81 & & Lanceolaria grayii & Lg04-01SUTO & Hiroshima, Japan & LC518989 & LC223990* & LC519062 \\
\hline U82 & & & Lg04-02SUTO & Hiroshima, Japan & LC518990 & LC223991* & \\
\hline U83 & & & Lg14-01SUTO & Gifu, Japan & LC518991 & LC223992* & \\
\hline U84 & & & $\operatorname{Lg} 14-2 \mathrm{f}$ & Gifu, Japan & LC518992 & LC223993* & LC519063 \\
\hline $\mathrm{U} 85$ & & & Lg-u3 & Shiga, Japan & LC518993 & LC519043 & \\
\hline U86 & & & Lg-u4 & Shiga, Japan & LC518994 & LC519044 & \\
\hline U87 & & Arconaia lanceolata & - & China & NC023955* & NC023955* & \\
\hline U88 & & & - & Dongting Lake, Hunan, China & & & MG595478* \\
\hline U89 & & Lanceolaria grayana & - & China & NC026686* & NC026686* & \\
\hline U90 & & Lanceolaria gladiola & - & Gan River, Jiangxi, China & & & MG595534* \\
\hline U91 & & Lepidodesma languilati & - & China & NC029491* & NC029491* & \\
\hline U92 & & & - & Gan River, Jiangxi, China & & & MG595544* \\
\hline U93 & & Aculamprotula tientsinensis & - & China & NC029210* & NC029210* & \\
\hline U94 & & & - & Nanxinxiang Gan River, Jiangxi, China & MF072504* & MF072511* & \\
\hline U95 & & Lamprotula coreana & - & Korea & NC026035* & NC026035* & \\
\hline U96 & & Lamprotula tortuosa & - & China & KC109779* & KC109779* & \\
\hline U97 & & Lamprotula caveata & - & Qinglan Lake, Jiangxi, China & & & MG595513* \\
\hline U98 & & Obovalis omiensis & Oo14-01 m & Gifu, Japan & LC518995 & LC223994* & \\
\hline U99 & & & Oo16-01SUTO & Kyoto, Japan & LC518996 & LC223995* & LC519064 \\
\hline U100 & & & Oo-u1 & Shiga, Japan & LC518997 & LC519045 & LC519065 \\
\hline U101 & & Ptychorhynchus pfisteri & - & China & KY067440* & KY067440* & \\
\hline U102 & & & - & Xiangyin, Hunan, China & & & MG595562* \\
\hline U103 & & Pronodularia japanensis & $\mathrm{Pj} 25-06$ & Sakai, Yamagata, Japan & LC518998 & LC223996* & \\
\hline U104 & & & Pj14-02f & Gifu, Japan & LC518999 & LC223997* & \\
\hline U105 & & & Pj14-05f & Gifu, Japan & LC519000 & LC223998* & LC519066 \\
\hline U106 & & & Pj04-01SUTO & Hiroshima, Japan & LC519001 & LC224001* & LC519067 \\
\hline $\mathrm{U} 107$ & & & Pj04-03SUTO & Hiroshima, Japan & LC519002 & LC224002* & \\
\hline $\mathrm{U} 108$ & & & $\mathrm{Pj}-\mathrm{k}$ & Sakurai, Nara, Japan & LC519003 & LC224019* & \\
\hline U109 & & Potomida littoralis & - & Mirandela Abreiro, Portugal & NC030073* & NC030073* & \\
\hline U110 & & & - & Cadiz, Spain & KP217871* & KP217981* & \\
\hline U111 & & & - & Cadiz, Spain & KP217873* & KP217983* & \\
\hline U112 & & & - & Cadiz, Spain & KP217872* & KP217982* & \\
\hline $\mathrm{U} 113$ & & Lamprotula leai & - & China & NC023346* & NC023346* & \\
\hline $\mathrm{U} 114$ & & & - & Qinglan Lake, Jiangxi, China & MF072503* & MF072510* & \\
\hline $\mathrm{U} 115$ & & Inversidens brandti & Ib14-01f & Gifu, Japan & LC519004 & LC224003* & LC519068 \\
\hline $\mathrm{U} 116$ & & & Ib14-02f & Gifu, Japan & LC519005 & LC224004* & LC519069 \\
\hline U117 & & Sinohyriopsis schlegeli & Hs21-02f & Lake Anenuma, Aomori, Japan & LC519006 & LC224005* & LC519070 \\
\hline U118 & & & Hs21-05f & Lake Anenuma, Aomori, Japan & LC519007 & LC224006* & LC519071 \\
\hline
\end{tabular}


Table 1. Continued.

\begin{tabular}{|c|c|c|c|c|c|c|c|}
\hline \# & Family & Species & Sample Ab. & Locality & $\begin{array}{l}\text { Accession } \\
\text { No. of } \\
\text { mitochondrial } \\
\text { COI DNA }\end{array}$ & $\begin{array}{l}\text { Accession } \\
\text { No. of } \\
\text { mitochondrial } \\
\text { 16S rDNA }\end{array}$ & $\begin{array}{l}\text { Accession } \\
\text { No. of } \\
\text { nuclear } \\
\text { 28S rDNA }\end{array}$ \\
\hline U119 & & Sinohyriopsis cumingii & - & China & HQ641406* & HQ641406* & \\
\hline U120 & & & - & China & HM347668* & HM347668* & \\
\hline U121 & & & - & Poyang Lake, Jiangxi, China & & & MG595611* \\
\hline U122 & & Lampsilis cardium & - & Illinois, USA & $\mathrm{KX} 713472^{*}$ & KX713226* & \\
\hline U123 & & Cristaria plicata & $\mathrm{Cp} 21-10 \mathrm{f}$ & Lake Anenuma, Aomori, Japan & LC519008 & LC224007* & \\
\hline U124 & & & $\mathrm{Cp} 21-11 \mathrm{f}$ & Lake Anenuma, Aomori, Japan & LC519009 & LC224008* & LC519072 \\
\hline U125 & & & Cp31-01fmg & Joetsu, Niigata, Japan & LC519010 & LC224009* & \\
\hline U126 & & & YAMAKARA 1 & Lake Yamanakako, Yamanashi, Japan & LC519011 & LC223968* & \\
\hline U127 & & & YAMAKARA 2 & Lake Yamanakako, Yamanashi, Japan & LC519012 & LC223969* & \\
\hline U128 & & & YAMAKARA 5 & Lake Yamanakako, Yamanashi, Japan & LC519013 & LC223971* & LC519073 \\
\hline U129 & & & YAMAKARA 6 & Lake Yamanakako, Yamanashi, Japan & LC519014 & LC223970* & \\
\hline U130 & & & - & Zhejiang, China & FJ986302* & FJ986302* & \\
\hline U131 & & & - & Gan River, Jiangxi, China & & & MG595485* \\
\hline U132 & & Sinanodonta lauta & $\mathrm{fk} 168$ & Ishikawa, Japan & LC519015 & LC224010* & LC519074 \\
\hline U133 & & & KONZAISYU E & Lake Biwa, Shiga, Japan & LC519016 & LC223967* & \\
\hline U134 & & & FUKUNUMA 22 & Minamisoma, Fukushima, Japan & LC519017 & LC223966* & LC519075 \\
\hline U135 & & Sinanodonta japonica & $\mathrm{fk} 20 \mathrm{f}$ & Kyoto, Japan & LC519018 & LC224011* & LC519076 \\
\hline U136 & & & $\mathrm{fk} 35 \mathrm{f}$ & Kushiro, Hokkaido, Japan & LC519019 & LC224012* & LC519077 \\
\hline U137 & & & $\mathrm{fk} 59 \mathrm{f}$ & Kagawa, Japan & LC519020 & LC519046 & \\
\hline U138 & & Sinanodonta calipygos & $\mathrm{fk} 221$ & Lake Biwa, Shiga, Japan & LC519021 & LC224013* & LC519078 \\
\hline U139 & & & $\mathrm{fk} 222$ & Lake Biwa, Shiga, Japan & LC519022 & LC519047 & LC519079 \\
\hline U140 & & Sinanodonta ogurae & fk156 & Yodo River, Osaka, Japan & LC519023 & LC224015* & LC519080 \\
\hline U141 & & & fk-o1 & Osaka, Japan & LC519024 & LC519048 & LC519081 \\
\hline $\mathrm{U} 142$ & & Sinanodonta woodiana & - & China & NC024943* & NC024943* & \\
\hline U143 & & Sinanodonta lucida & - & Asian continent & NC026673* & NC026673* & \\
\hline U144 & & Sinanodonta angula & - & Anren County, Hunan, China & & & MG595576* \\
\hline U145 & & Anemina arcaeformis & fk63f & Kagawa, Japan & LC519025 & LC224014* & LC519082 \\
\hline U146 & & & $\mathrm{fk} 90 \mathrm{f}$ & Ishikari, Hokkaido, Japan & LC519026 & LC519049 & LC519083 \\
\hline U147 & & & - & Asian continent & KF667530* & KF667530* & \\
\hline U148 & & & - & Qinglan Lake, Jiangxi, China & & & MG595464* \\
\hline U149 & & Anemina euscaphys & - & China & NC026792* & NC026792* & \\
\hline $\mathrm{U} 150$ & & Pletholophus tenuis & Pt43-02 & Munakata, Fukuoka, Japan & LC519027 & LC224017* & LC519084 \\
\hline U151 & & & Pt43-03 & Munakata, Fukuoka, Japan & LC519028 & LC224018* & LC519085 \\
\hline U152 & & Alasmidonta varicosa & - & Connecticut River, Croyden, Brook, USA & MG938673* & MG938673* & \\
\hline U153 & & Acuticosta chinensis & - & Gan River, Jiangxi, China & & & MG595450* \\
\hline U154 & & Aculamprotula tortuosa & - & Qinglan Lake, Jiangxi, China & & & MG595443* \\
\hline U155 & & Alasmidonta heterodon & - & USA & $\mathrm{NC} 037431^{*}$ & NC037431* & \\
\hline U156 & & Lasmigona compressa & - & USA & HM856638* & HM856638* & \\
\hline U157 & & Pyganodon grandis & - & USA & FJ809754* & FJ809754* & \\
\hline $\mathrm{U} 158$ & & Utterbackia imbecillis & - & USA & HM856637* & HM856637* & \\
\hline U159 & & Utterbackia peninsularis & - & USA & HM856636* & HM856636* & \\
\hline $\mathrm{U} 160$ & & Anodonta anatina & - & Poland & NC022803* & NC022803* & \\
\hline U161 & & Anodonta cygnea & - & Germany & NC036488* & NC036488* & \\
\hline U162 & & Schistodesmus lampreyanus & - & Gan River, Jiangxi, China & & & MG595569* \\
\hline U163 & & Solenaia oleivora & - & Poyang Lake, Jiangxi, China & & & MG595618* \\
\hline U164 & Hyriidae & Hyridella australis & BivAToL-378 & Australia, New South Wales & KX713467* & KX713224* & \\
\hline U165 & & Velesunio ambiguus & - & Australia, New South Wales & KC429106* & KC429263* & \\
\hline U166 & & Triplodon corrugatus & BivAToL-380 & Peru & KX713505* & KX713262* & \\
\hline U167 & Mycetopodidae & Anodontites elongata & BivAToL-323 & Peru & KX713444* & KX713190* & \\
\hline U168 & & Lamproscapha ensiformis & BivAToL-382 & Peru & KX713471* & KX713225* & \\
\hline U169 & Etheriidae & Etheria elliptica & BivAToL-404 & Zambia & KX713462* & KX713219* & \\
\hline U170 & Iridinidae & Aspatharia pfeifferiana & BivAToL-330 & Zambia & $\mathrm{KC} 429107^{*}$ & KC429264* & \\
\hline U171 & & Chambardia wahlbergi & BivAToL-405 & Zambia & KX713448* & KX713202* & \\
\hline U172 & & Mutela hargeri & BivAToL-401 & Zambia & KX713482* & KX713237* & \\
\hline U173 & Trigoniidae & Neotrigonia margaritacea & - & Australia & KX713243* & KX713243* & \\
\hline U174 & & Neotrigonia lamarckii & - & Queensland, Australia & KC429262* & KC429262* & \\
\hline U175 & & & - & Queensland, Australia & & & AM779652* \\
\hline
\end{tabular}


using nested PCR. The first set of PCR reactions was conducted using the sense and antisense primers FWCO1-2F, 5'-CAA ACC TAT CTG GAT AAT CAG AAT ACC GAC GAG G-3' and FWCO2-2R, 5'-TGA GCT TTT GGG GTC AAT TAG GGT TTC A-3' under the following conditions: initial denaturation at $95^{\circ} \mathrm{C}$ for $3 \mathrm{~min} ; 45$ cycles of denaturation at $95^{\circ} \mathrm{C}$ for $45 \mathrm{sec}$, annealing at $60^{\circ} \mathrm{C}$ for $10 \mathrm{~min}$, and extension at $72^{\circ} \mathrm{C}$ for $1 \mathrm{~min}$; followed by a final extension at $72^{\circ} \mathrm{C}$ for $7 \mathrm{~min}$. The second set of PCR reactions was performed using the sense and antisense primers HCO2198-1F, 5'-TAC ACT TCA GGA TGA CCA AAA AAC CA-3' and LCO1490-1R, 5'-GGT GAT TGT GTT CTA CTA ATC ATA AGG ATA TTG G-3' under the following conditions: initial denaturation at $95^{\circ} \mathrm{C}$ for $3 \mathrm{~min}$; 35 cycles of denaturation at $95^{\circ} \mathrm{C}$ for $45 \mathrm{sec}$, annealing at $60^{\circ} \mathrm{C}$ for $3 \mathrm{~min}$, and extension at $72^{\circ} \mathrm{C}$ for $30 \mathrm{sec}$; followed by a final extension at $72^{\circ} \mathrm{C}$ for $7 \mathrm{~min}$.

PCR products for the nuclear $28 \mathrm{~S}$ and mitochondrial COI were purified using a QIAquick ${ }^{\circledR}$ PCR Purification Kit (QIAGEN GmbH, Hilden, Germany). Sequence reactions were performed using a GenomeLab ${ }^{\text {TM}}$ DTCS-Quick Start Kit (Beckman Coulter Inc., California, USA) and the same primers for the final PCR under the following conditions: 30 cycles of denaturation at $96^{\circ} \mathrm{C}$ for $20 \mathrm{sec}$, annealing at $50^{\circ} \mathrm{C}$ for $20 \mathrm{sec}$, and extension at $60^{\circ} \mathrm{C}$ for $4 \mathrm{~min}$. Direct sequencing of the double-stranded PCR products was performed using a CEQ ${ }^{\mathrm{TM}}$ 2000XL DNA Analysis system (Beckman Coulter Inc., California, USA) according to the manufacturer's instructions. Mitochondrial 16S rDNA was amplified and sequenced as described previously (Sano et al. 2017).

\section{Phylogenetic analysis}

Alignments of nuclear 28S rDNA, mitochondrial COI, and 16S rDNA sequences were performed using MUSCLE v3.8 (Edgar 2004). The results were confirmed by visual inspection using MEGA 6.0 (Tamura et al. 2013). Then, trimAl v1.4 (Capella-Gutiérrez et al. 2009) was used to remove regions of the 16S rDNA and 28S rDNA aligned sequences unsuitable for phylogenetic analysis. We constructed trees using 413-bp 28S rDNA. We also used 937bp concatenated COI DNA+16S rDNA sequences, including 532-bp COI DNA and 405-bp 16S rDNA, for the construction of trees.

Bayesian (BI) trees were constructed using MrBayes5d version 3.1.2.2012.12.13 (Ronquist et al. 2012; Tanabe 2008), based on a model evaluation performed using PartitionFinder v2.1.1 (Lanfear et al. 2016) (Table 2). The Markov chain Monte Carlo (MCMC) lengths for nuclear and mitochondrial DNA were $6 \times 10^{6}$ and $3 \times 10^{7}$ generations, respectively, and we sampled the chain every 1,000 generations. MCMC convergence was assessed by calculating the potential scale reduction factor, and the first 601 and 3,001 generations were discarded for nuclear and mitochondrial DNA, respectively. The program Tracer v. 1.6 (Rambaut et al. 2013) was used to evaluate MCMC chain
Table 2. Partitioning schemes and best fit models identified using PartitionFinder for the nuclear and combined mitochondrial dataset. " $+\mathrm{X}$ " was added when base frequencies were estimated.

\begin{tabular}{lcl}
\hline $\begin{array}{l}\text { Partition } \\
\text { contents }\end{array}$ & $\begin{array}{c}\text { Model of sequencing } \\
\text { evolution: MrBayes } \\
\text { and IQ-TREE }\end{array}$ & $\begin{array}{c}\text { Model of sequencing } \\
\text { evolution: BEAST }\end{array}$ \\
\hline $28 \mathrm{~S}$ & $\mathrm{GTR}+\mathrm{G}$ & - \\
COI 1st & $\mathrm{GTR}+\mathrm{I}+\mathrm{G}$ & $\mathrm{TRN}+\mathrm{I}+\mathrm{G}+\mathrm{X}$ \\
COI 2nd & $\mathrm{TIM}+\mathrm{I}$ & $\mathrm{GTR}+\mathrm{I}+\mathrm{X}$ \\
COI 3rd & $\mathrm{GTR}+\mathrm{G}$ & $\mathrm{GTR}+\mathrm{G}+\mathrm{X}$ \\
$16 \mathrm{~S}$ & $\mathrm{GTR}+\mathrm{I}+\mathrm{G}$ & $\mathrm{GTR}+\mathrm{I}+\mathrm{G}+\mathrm{X}$ \\
\hline
\end{tabular}

convergence and to compute marginal posterior distributions of parameters, after the removal of $10 \%$ of the chain as burn-in. In the case of the mitochondrial DNA analysis, when the temperature was the initial setting, the likelihood did not cease, so the temperature was set to 0.15 . A maximum-likelihood (ML) tree was constructed using IQ-TREE (Nguyen et al. 2014) based on a model evaluation performed using PartitionFinder v2.1.1 (Lanfear et al. 2016) (Table 2). The tree reliability was evaluated by generating 1,000 bootstrap replicates.

Recent studies have published evidence that could be useful for more accurately estimating the divergence times of unionoid mussels. Fossil records were reported (Huang et al. 2018) and molecular clock rates based on geographic events were estimated for unionoid mussels (Froufe et al. 2016). Fossil records are based primarily on shell morphology. However, because previous studies have shown that there are cases in which the shell morphology and molecular lineage of unionoid mussels are not consistent (Pfeiffer \& Graf 2013), there is a possibility of overestimating the divergence time based on fossil records. Thus, we conducted divergence time estimation based on the molecular clock rates in addition to fossil record-based estimation. Both nuclear and mitochondrial sequences of the same individuals have rarely been published, and much more sequence data have been published for mitochondrial genes than for nuclear genes. Therefore, we estimated the divergence time using BEAST2 v2.4.4. (Bouckaert et al. 2014) without the nuclear sequence dataset. The Bayesian inference was based on the substitution rates estimated using geological events (tree prior $=$ Yule process; ngen $=3.6 \times 10^{8} ;$ samplefreq $=1,000$; clock models $=$ uncorrelated lognormal relaxed clock) and based on molecular clock node calibrations estimated by fossil records (tree prior $=$ Yule process; ngen $=5.4 \times 10^{8}$; samplefreq $=1,000$; clock models $=$ uncorrelated lognormal relaxed clock). When we used substitution rates estimated based on geological events, we made estimates using a substitution rate for COI of $0.265 \pm 0.06 \%$ /million years, which was reported by Froufe et al. (2016). Froufe et al. (2016) examined the impact of the Strait of Gibraltar, which appeared around 5.33 Mya (Krijgsman et al. 1999), on the diversification of Unio species. Using the 
results of this study, they calculated the substitution rates, which are currently the most reliable within Unionoida. In contrast, when we used molecular clock node calibrations estimated based on fossil records, the constraints, as indicated by Huang et al. (2018), were as follows: (1) The tree root height of Palaeoheterodonta (normal distribution prior, mean $=475$, stdev $=2$ ); (2) Margaritiferidae + Unionidae (exponential prior, $\min =230 \mathrm{Ma}$, lambda $=30$ ); (3) Unionidae (exponential prior, $\min =152 \mathrm{Ma}$, lambda $=20$ ); (4) The most recent common ancestor (MRCA) of M. falcata-M. laevis (exponential prior, $\min =46 \mathrm{Ma}$, lambda $=12.5$ ); (5) The MRCA of $M$. dahurica-M. margaritifera (exponential prior, $\min =34 \mathrm{Ma}$, lambda=9.3); and (6) The MRCA of $M$. marocana-M. auricularia (exponential prior, $\min =35$ Ma, lambda=9.5). Huang et al. (2018) estimated the divergence time, mainly within Margariferidae, using fossils of the early Ordovician genus Noradonta and the oldest fossil Shifangella assigned to Margariferidae. We examined convergence and effective sample size (ESS) using Tracer v. 1.6 (Rambaut et al. 2013). Substitution models of each partition were selected from the available evolutionary models of BEAST2 v2.4.4 (Bouckaert et al. 2014) using PartitionFinder v2.1.1 (Lanfear et al. 2016) (Table 2). We used Neotrigonia (KX713243, KC429262, and AM779652) sequences as the outgroup (Table 1).

\section{Results}

Fig. 1 shows the phylogenetic relationships among Japanese unionoid mussels based on the sequences of their nuclear $28 \mathrm{~S}$ rDNA $(413 \mathrm{bp})$. There were 145 variable and 103 informative sites in the $28 \mathrm{~S}$ tree. The topologies depicted by the BI trees were essentially identical to those depicted by the ML tree. Japanese unionoid mussels were divided into two clades corresponding to the two families Margaritiferidae and Unionidae. In Unionidae, there were two clades corresponding to the subfamilies Unioninae, and Gonideinae. The resolution of distal clades, however,

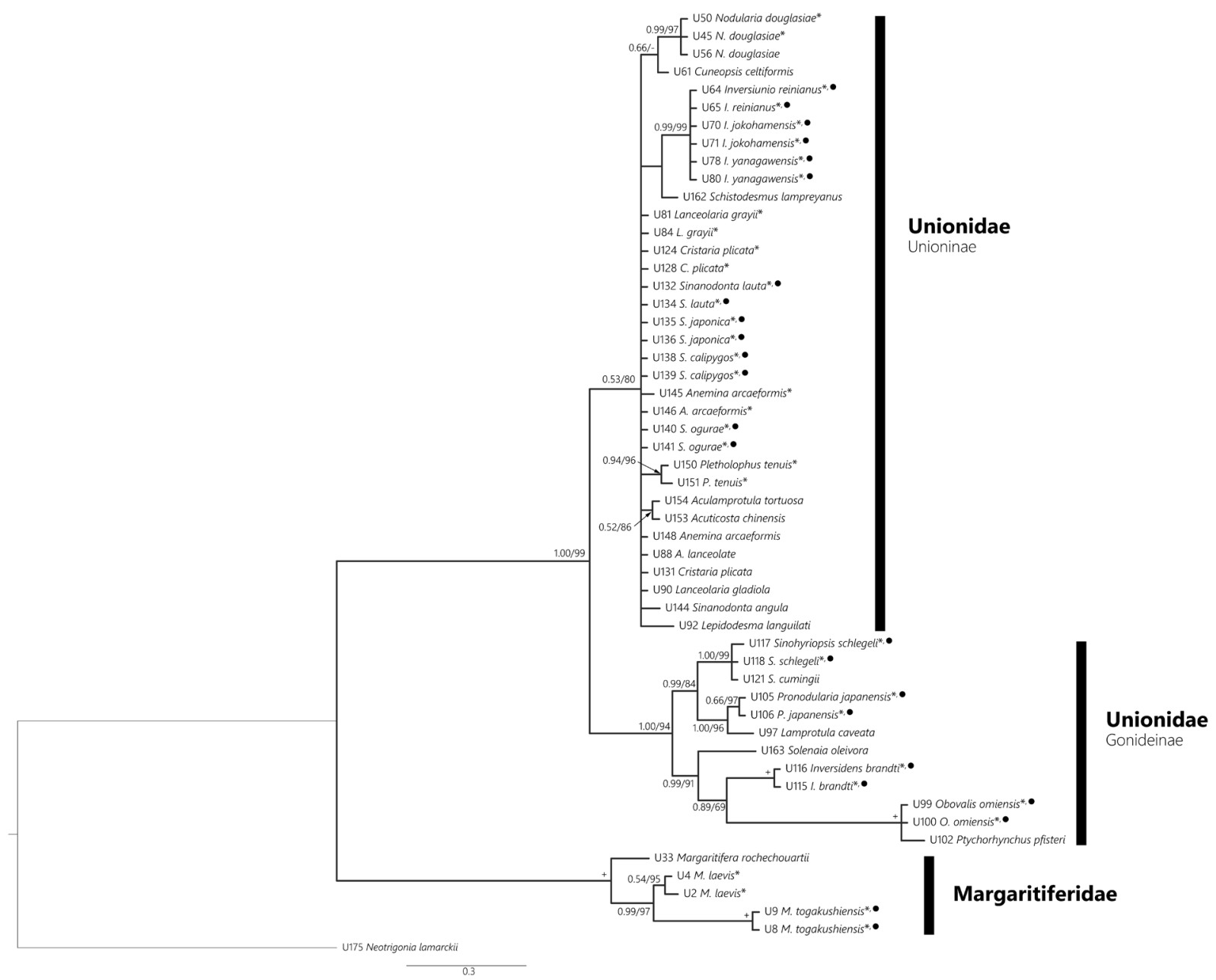

Fig. 1. Phylogeny of Japanese unionoid mussels based on nuclear $28 \mathrm{~S}$ rDNA. This phylogenetic tree was constructed based on 413 bp of the nuclear 28S gene. Neotrigonia was used as an outgroup. Bayesian inference (BI) posterior probabilities (left) and maximum-likelihood (ML) bootstrap values (right) are specified near the relevant nodes. The operational taxonomic units (OTUs) accompanied by “*” live in the Japanese archipelago. " $\bullet$ indicates the endemic species in Japan. Nodes, supported by a probability of $1.00 / 100$, were written as “+”. 


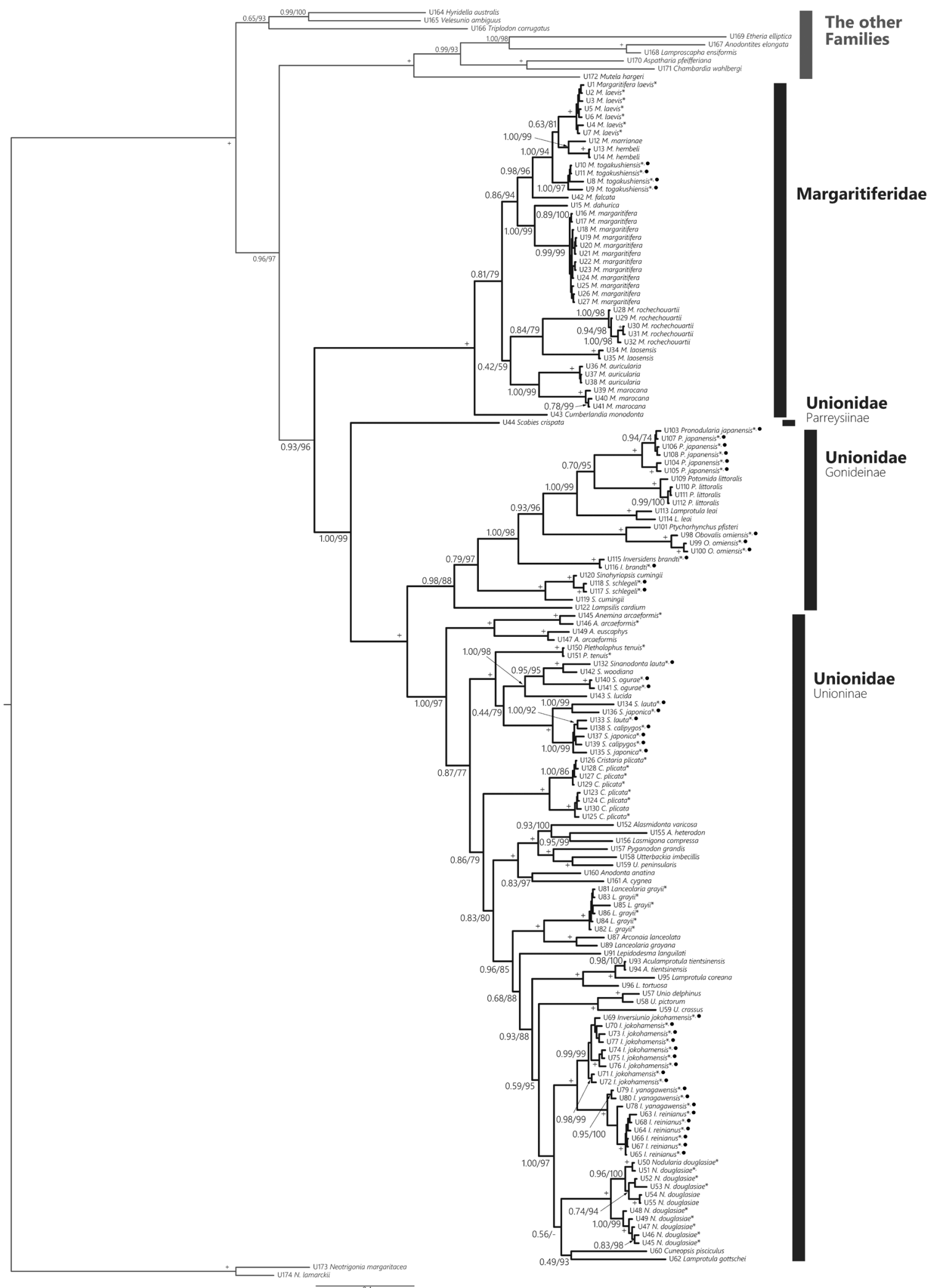

Fig. 2. Phylogeny of the Japanese unionoid mussels based on mitochondrial COI and 16S rDNA. This phylogenetic tree was constructed based on a total of $937 \mathrm{bp}$ of mitochondrial COI and 16S genes. Neotrigonia was used as an outgroup. Bayesian inference (BI) posterior probabilities (left) and maximum-likelihood (ML) bootstrap values (right) are specified near the relevant nodes. The operational taxonomic units (OTUs) accompanied by “*” live in the Japanese archipelago. "•" indicates the endemic species in Japan. Nodes, supported by a probability of $1.00 / 100$, were written as “+”. 


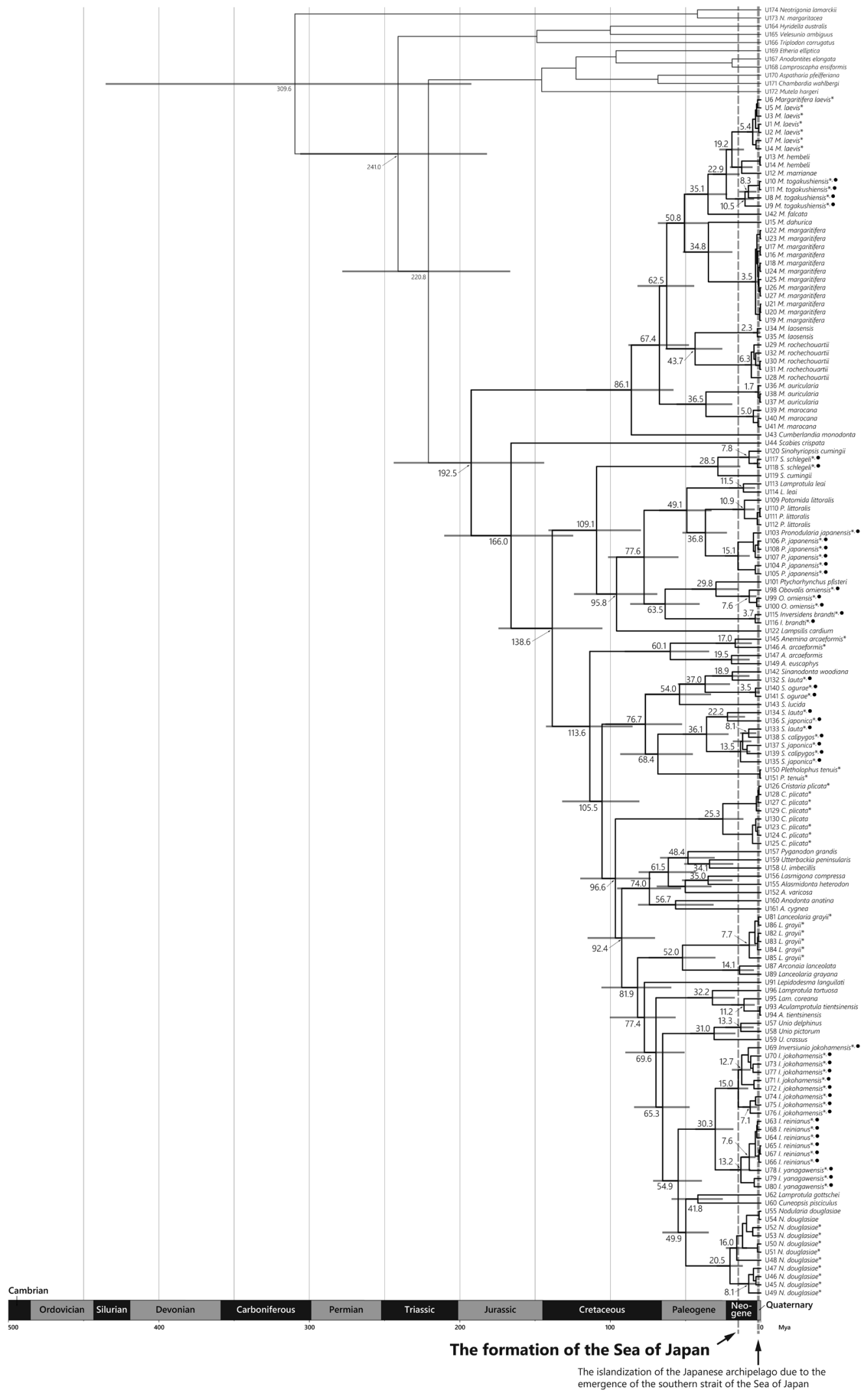

Fig. 3. Time estimation based on substitution rates based on geological events. This phylogenetic tree was constructed based on a total of 937 bp of mitochondrial COI and 16S genes. Neotrigonia was used as an outgroup. The gray node bar represents the $95 \%$ confidence interval for the divergence time. The operational taxonomic units (OTUs) accompanied by “*” live in the Japanese archipelago. "•” indicates the endemic species in Japan. 


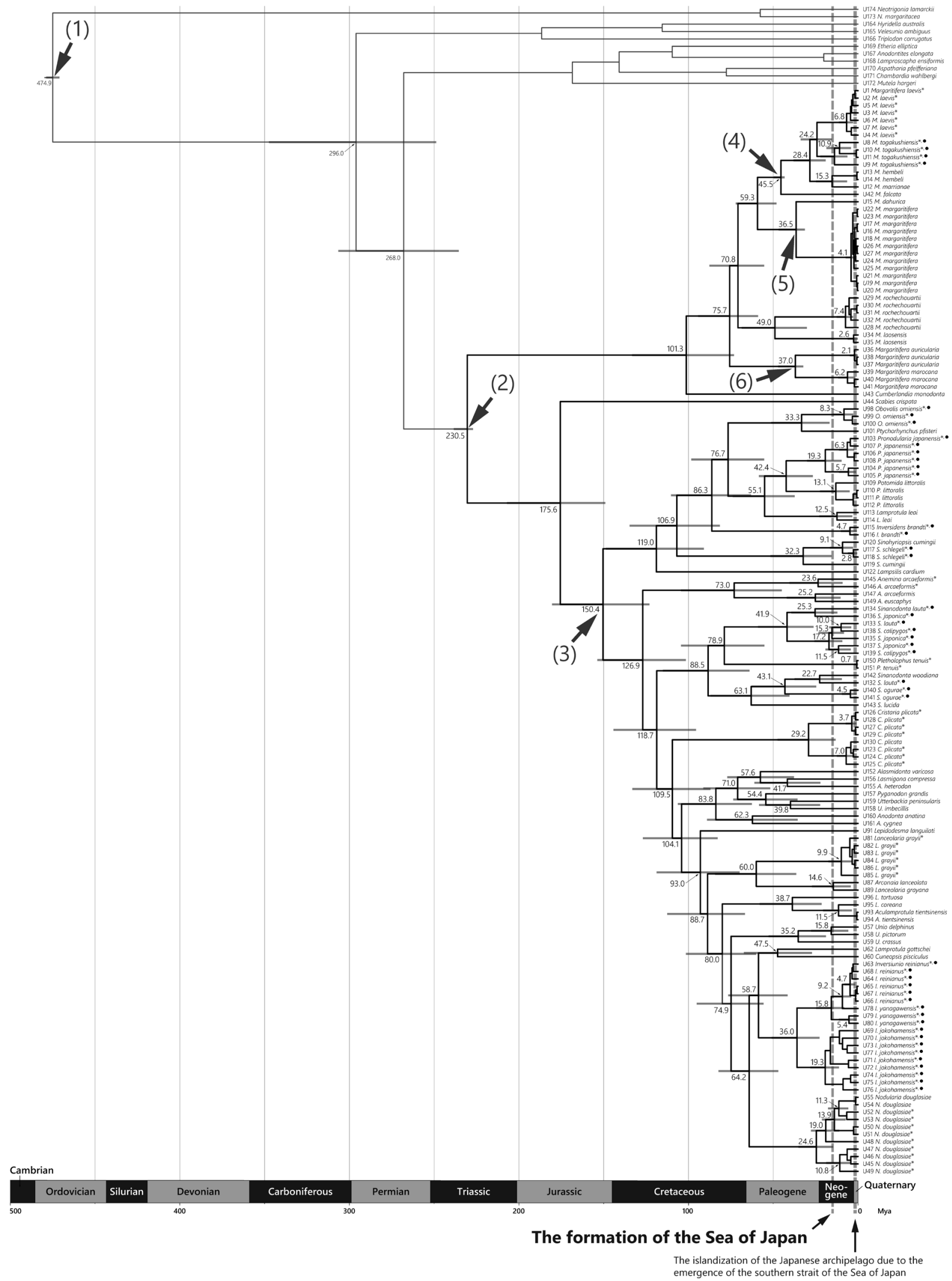

Fig. 4. Time estimation based on fossil records. This phylogenetic tree was constructed based on a total of $937 \mathrm{bp}$ of mitochondrial COI and $16 \mathrm{~S}$ genes. Neotrigonia was used as an outgroup. The gray node bar represents the $95 \%$ confidence interval for the divergence time. The operational taxonomic units (OTUs) accompanied by “*” live in the Japanese archipelago. "•" indicates the endemic species in Japan. The numbers in parentheses indicate the locations of the constraints used in the analysis. 
was poor, and detailed phylogenetic relationships among species and among genera could not be obtained.

Fig. 2 shows the phylogenetic relationships of Japanese unionoids based on combined sequences $(937 \mathrm{bp}$ ) of the $16 \mathrm{~S}$ rRNA and COI genes. There were 264 variable and 249 informative sites in the COI and 283 variable and 227 informative sites in the 16S rDNA. The topologies depicted by the BI trees were essentially identical to those depicted by the ML tree. We presented the $16 \mathrm{~S}$ rDNA tree in our previous study (Sano et al. 2017) and revised it here by adding the newly collected specimens of Nodularia douglasiae, Inversiunio jokohamensis, I. reinianus, Lanceolaria grayii, Anemina arcaeformis, Sinanodonta ogurae, S. japonica, S. calipygos, Obovalis omiensis, Margaritifera laevis, and M. togakushiensis.

Japanese unionoid mussels were divided into two clades corresponding to two families, the Margaritiferidae and Unionidae. In Unionidae, there were three clades corresponding to the subfamilies Parreysiinae, Unioninae, and Gonideinae. Most clades corresponding to each genus were formed with high statistical support. The only exception was Sinanodonta $(0.44 / 79)$.

Most species living in the Japanese archipelago formed clades with robust statistical supports, even though the specimens of some species were collected from distant localities. Based on Kondo (2008, 2015), species living in the Japanese archipelago can be broadly divided into two types: a) species that are not endemic to the Japanese archipelago and are found on both the Asian continent and Japan, and b) species that are endemic to Japan and occur only in the Japanese archipelago. The above type a) species include M. laevis, A. arcaeformis, Pletholophus tenuis, Cristaria plicata, L. grayii, and $N$. dougulasiae. All these species formed their own clade, except for A. arcaeformis. Type b) species include M. togakushiensis, Pronodularia japanensis, O. omiensis, Inversidens brandti, Sinohyriopsis schlegeli, S. lauta, S. japonica, S. calipygos, S. ogurae, I. jokohamensis, I. yanagawensis, and I. reinianus. All these species formed their own clade, except for S. lauta, S. japonica, S. calipygos, and I. yanagawensis. Three Sinanodonta species, S. lauta, S. calypigos, and S. japonica, did not form their own clades and exhibited complicated relationships with $S$. ogurae.

Two types of divergence time estimations were made: one based on evolutionary rates (Fig. 3) and another based on fossil records (Fig. 4). The divergence times among the major clades were older for those estimated by fossil records than for those by molecular clock rates, but their $95 \%$ confidence intervals were mostly overlapped. When we assumed that the Japanese archipelago started to develop at the time of the formation of the Sea of Japan (Figs. 3 and 4); 10 of the 12 endemic Japanese species were found to diverge before the formation of the Japanese archipelago (M. togakushiensis, P. japanensis, O. omiensis, I. brandti, S. lauta, S. japonica, S. calipygos, S. ogurae, I. jokohamensis, and I. yanagawensis). In contrast, two species, $S$. schlegeli and I. reinianus, may have diverged after the formation of the Japanese archipelago.

\section{Discussion}

In this study, we sequenced all 18 species of the order Unionoida from Japan and examined the evolutionary history and species diversity of the order before and after the formation of the Japanese archipelago using published data for the Asian continent. The results revealed that nuclear sequences did not have as much phylogenetic information as mitochondrial sequences in the case of Unionoida. Nuclear 28S rDNA was suitable for estimating family-level and subfamily-level phylogenetic relationships, but not for estimating lower-order phylogenetic relationships (Fig. 1). Thus, the results based on mitochondrial DNA are discussed.

Unionoid mussels living in the Japanese archipelago were found to be highly endemic. Detailed genetic analysis revealed that most of the 12 species endemic to the Japanese archipelago formed monophyletic groups and differentiated independently (Fig. 2). In contrast, our results also confirmed that some species of the genera Sinanodonta and Inversiunio showed morphological and molecular phylogenetic inconsistencies, and Sinanodonta lauta, S. japonica, S. calipygos, and Inversiunio yanagawensis were not found to form their own clade upon molecular phylogenetic analysis. In general, diverse morphologies are found within several mollusk genera and species, often interfering with species recognition based on the concept of reproductive isolation (Pfeiffer \& Graf 2013; Hirano et al. 2015). Furthermore, it is suggested that species within a genus may have undergone plastic changes in morphology as they adapted to their habitat, because each of these genera formed a separate monophyletic group. All species of the genus Inversiunio are endemic to Japan: i) I. jokohamensis is found mainly east of Lake Biwa; ii) I. yanagawensis is found mainly west of Lake Biwa; and iii) I. reinianus is found only in Lake Biwa (Kondo 2008, 2015). Judging from the phylogenetic trees in this study, it is suggested that the endemic species of Lake Biwa, I. reinianus, was possibly differentiated from I. yanagawensis. Furthermore, while I. yanagawensis formed a paraphyletic group, the clade of I. reinianus $+I$. yanagawensis was supported with high confidence, suggesting that a rapid evolution of morphology has occurred in Lake Biwa. Phylogenetic trees based on the nuclear $28 \mathrm{~S}$ rDNA did not have sufficient information to elucidate the differentiation process within the genus Inversiunio. The results of this study indicate that morphological classification and phylogenetic relationships are not necessarily concordant, as is the case for Sinanodonta and Inversiunio. It is unclear whether this is due to a plastic change in morphology or because the molecular markers used in this study do not correctly reflect the differentiation among morphological species. Therefore, it is necessary to analyze a large amount of genomic informa- 
tion in combination with morphological re-examination to clarify this issue.

The Japanese archipelago is geographically close to the Asian continent, and a lot of lineages of its endemic fauna are closely related to continental lineages. These Japanese lineages may have originated on the Asian continent. Cristaria plicata and Nodularia douglasiae are distributed widely in East Asia, while they could not be genetically separated between Japan and the Asian continent. This can be explained by "back dispersal" (Tojo et al. 2017) from the Japanese archipelago to the Asian continent. This may also be explained by artificial genetic contamination, as is observed between Japanese Sinohyriopsis schlegeli and Chinese $S$. cumingii (Shirai et al. 2010). However, to address this issue, further genetic research is needed.

The Japanese archipelago is geographically close to the Asian continent; however, the margaritiferid mussels in the archipelago are genetically close to species associated with the North American continent. The larvae of unionoid mussels are known to be salt-tolerant while being parasitic to host fishes (Itoh et al. 2017), suggesting that margaritiferid mussels may have invaded the Japanese archipelago from North America by dispersion through ocean currents. The parasitism period of glochidia on fishes has not yet been fully elucidated, but is reported to be approximately twenty five days for $S$. schlegeli (Hatano \& Ishizaki 2016) and approximately ten days for $N$. douglasiae (Itoh 2013), and it is believed that unionoid mussels are often parasitic for more than one week. It is also known that the lower the water temperature, the longer the parasitism period (Hurukawa et al. 1965). Therefore, it is considered that the parasitism period becomes longer in environments with low water temperature, such as the Bering Sea, the Sea of Okhotsk, and the Sea of Japan. In future research, it is necessary to examine the parasitism period of glochidia on fishes in detail to investigate whether or not freshwater mussels can disperse across the sea. The larval hosts of the Margaritifera in Japan and western North America are the salmonids Oncorhynchus and Salmo (Taylor \& Uyeno 1965). The distribution ranges of these mussels overlap with those of salmonids. Therefore, it is possible that the salmonids carried the glochidia of these mussels from North America to the Japanese archipelago.

The estimations of the divergence time suggest that the unionoid species that are now widely distributed in East Asia diversified on the Asian continent long before the formation of the Japanese archipelago (Figs. 3, 4). The diversification of most Japanese endemic species began before the Japanese archipelago separated from the Asian continent because the Sea of Japan was formed 15 million years ago. Although taxon sampling of continental species might be insufficient to estimate the exact age, this study provides the most reliable estimation for the divergence time of the East Asian unionoid mussels so far; all of the genera and their representative species of the East Asian region described by Lopes-Lima et al. (2017) are included in this study. An alternative hypothesis is that continental species that are phylogenetically closely related to Japanese extant species have already gone extinct. Previous studies on insect fauna have shown that the formation of the Japanese archipelago caused high species diversity in Japan (Gamboa et al. 2019). Future surveys using fossil records from the Asian continent are needed to address this issue. However, unionoid mussels have proportionally many more endemic species than other organisms such as insects, suggesting that unionoid mussels have a lower dispersal ability than other organisms. Adult unionoid mussels have a lower dispersal ability than other freshwater organisms, and their passive dispersal ability in the larval stage is also limited. The limited passive dispersal ability of unionoid mussels may reflect the low dispersal ability of the fishes that carry parasitic larvae of unionoid mussels. Because of their low dispersal ability, it is most likely that the diversification of Japanese unionoid mussels had started long before the formation of the Japanese archipelago.

Likewise, mussels diverged at earlier times than freshwater fishes and snails. Japanese planorbid snails diverged 6.5 million years ago (mya), whereas Japanese cyprinid fishes diverged around 18 mya (Saito et al. 2018b; JangLiaw et al. 2019). In contrast, species of the unionoid genus endemic to Japan were found to have diverged earlier than approximately 60 mya (for example, 63.5 and 86.3 mya for Inversidens, as shown in Fig. 3 and in Fig. 4, respectively). This indicates that mussels are genetically more unique than other freshwater organisms.

In contrast, some species may have diverged after the formation of the Sea of Japan (S. schlegeli and I. reinia$n u s)$. These species are endemic to Lake Biwa, one of the ancient lakes in Japan. Since Lake Biwa began to form about 4 mya (Satoguchi 2012), the values of the divergence time estimations obtained in this study are within the estimated range of time in which S. schlegeli and I. reinianus adaptively differentiated to the habitat of Lake Biwa. Two other endemic species of Lake Biwa are known: $S$. calipygos and $S$. ogurae. The results of this study showed that these two species diverged before the formation of the Japanese archipelago. However, S. ogurae may have had an excessively large divergence times because of the extinction of related species. In fact, fossils of Kobiwakodonta nakajimai, which is considered to be a closely related extinct species of $S$. ogurae, have been excavated from the Kobiwako Group (Nakano et al. 2003). In addition, it was shown that $S$. calipygos is a polyphyletic group (Fig. 2). This result suggests the parallel morphological evolution of S. calipygos; however, the cause for this is not yet fully understood and may be the standing variation or low resolution of the trees.

Most freshwater creatures face high risks of extinction (Strayer et al. 2004; Walker et al. 2014). Unionoid mussels are especially likely to suffer the impacts of anthropogenic activities because of their poor dispersal ability and symbiosis with freshwater fishes during their development. This 
study could contribute to the promotion of future taxonomic revisions and morphological and molecular phylogenetic and phylogeographic studies, as well as the proposal of scientifically refined plans to conserve unionoids.

\section{Acknowledgements}

We express our sincere appreciation to Mr. Osamu Inaba, Mr. Koji Fujimoto, Dr. Shigefumi Kanao, and Mr. Shigeharu Togashi for their assistance in collecting unionoid specimens. Thanks are also extended to Dr. Takahiro Hirano, Dr. Daishi Yamazaki, Dr. Kazuki Kimura, Dr. Naoto Hanzawa, Dr. Jun Nakajima, Dr. Mitsunori Nakano, Dr. Daisuke Tanaka, Mr. Toshio Nakajima, Mr. Akihiro Kuwahara, Mr. Tomoki Hirose, Mr. Tadashi Okoba, Mr. Akimasa Hattori, and Mr. Bin Ye for generously supplying us with Japanese unionoid specimens. We would also like to thank Dr. Tomoki Seo, Dr. Keiji Matsuoka, Dr. Yasufumi Fujimoto, Dr. So Ishida, and Mr. Yuuki Yashima for providing useful information on mussels. We thank the two anonymous reviewers for their careful reading of our manuscript and their many insightful comments and suggestions. This study was supported in part by Research Grant for Young Scholars funded by Yamanashi Prefecture.

\section{References}

Arbogast BS, Kenagy GJ (2001) Comparative phylogeography as an integrative approach to historical biogeography. J Biogeogr 28: 819-825.

Boag DA (1986) Dispersal in pond snails: potential role of waterfowl. Can J Zool 64: 904-909.

Bouckaert R, Heled J, Kühnert D, Vaughan T, Wu CH, Xie D, Suchard MA, Rambaut A, Drummond AJ (2014) BEAST 2: a software platform for Bayesian evolutionary analysis. PLoS Comput Biol 10: e1003537.

Capella-Gutiérrez S, Silla-Martínez JM, Gabaldón T (2009) trimAl: a tool for automated alignment trimming in large-scale phylogenetic analyses. Bioinfomatics 25: 1972-1973.

Ceballos G, Brown JH (1995) Global patterns of mammalian diversity, endemism, and endangerment. Conserv Biol 9: 559568.

Cronin TM, Kitamura A, Ikeya N, Watanabe M, Kamiya T (1994) Late Pliocene climate change 3.4-2.3 Ma: paleoceanographic record from the Yabuta Formation, Sea of Japan. Palaeogeogr Palaeoclimatol Palaeoecol 108: 437-455.

Edgar RC (2004) MUSCLE: multiple sequence alignment with high accuracy and high throughput. Nucleic Acids Res 32: 1792-1797.

Fonseca CR (2009) The silent mass extinction of insect herbivores in biodiversity hotspots. Conserv Biol 23: 1507-1515.

Froufe E, Gonçalves DV, Teixeira A, Sousa R, Varandas S, Ghamizi M, Zieritz A, Lopes-Lima M (2016) Who lives where? Molecular and morphometric analyses clarify which Unio species (Unionida, Mollusca) inhabit the southwestern Palearctic. Org Diversity Evol 16: 597-611.
Gamboa M, Muranyi D, Kanmori S, Watanabe K (2019) Molecular phylogeny and diversification timing of the Nemouridae family (Insecta, Plecoptera) in the Japanese Archipelago. PLoS One 14: e0210269.

Gao Y, Wang SY, Luo J, Murphy RW, Du R, Wu SF, Zhu CL, Li Y, Poyarkov AD, Nguyen SN, Luan PT, Zhang YP (2012) Quaternary palaeoenvironmental oscillations drove the evolution of the Eurasian Carassius auratus complex (Cypriniformes, Cyprinidae). J Biogeogr 39: 2264-2278.

Green AJ, Figuerola J (2005) Recent advances in the study of long - distance dispersal of aquatic invertebrates via birds. Diversity Distrib 11: 149-156.

Hamano Y, Tosha T (1985) Movement and paleomagnetism in Northeast Japan. Kagaku 55: 476-483. (in Japanese)

Hatano M, Ishizaki D (2016) Feeding of juvenile freshwater pearl mussel Hyriopsis schlegeli. Venus 74: 3-4. (in Japanese with English summary)

Hirano T, Saito T, Chiba S (2015) Phylogeny of freshwater viviparid snails in Japan. J Molluscan Stud 81: 435-441.

Hirano T, Saito T, Tsunamoto Y, Koseki J, Ye B, Do VT, Miura O, Suyama Y, Chiba S (2019) Enigmatic incongruence between mtDNA and nDNA revealed by multi-locus phylogenomic analyses in freshwater snails. Sci Rep 9: 6223.

Huang XC, Wu RW, An CT, Xie GL, Su JH, Ouyang S, Zhou $\mathrm{CH}, \mathrm{Wu}$ XP (2018) Reclassification of Lamprotula rochechouartii as Margaritifera rochechouartii comb. nov. (Bivalvia: Margaritiferidae) revealed by time-calibrated multi-locus phylogenetic analyses and mitochondrial phylogenomics of Unionoida. Mol Phylogenet Evol 120: 297-306.

Hurukawa M, Kobayahsi Y, Hiratsuka T (1965) Research on artificial proliferation of Hyriopsis schlegeli-II. Bull Fish Exp Stn Shiga Prefect 18: 59-65. (in Japanese)

Itoh T, Oda N, Maruyama T (2003) Availability of host fish for glochidia of the freshwater unionid mussel Pronodularia japanensis. Jpn J Ecol 53: 187-196. (in Japanese with English summary)

Itoh T (2013) Two alien species, largemouth bass and tadpole of American bullfrog, are unsuitable hosts for the glochidia of the freshwater unionid mussel Unio douglasiae nipponensis in the Kanto area, Central Japan. Venus 71, 1-2. (in Japanese with English summary)

Itoh T, Kakino W, Kitano T, Kohno H (2017) Salinity tolerance of six adults (Inversiunio jokohamensis, Hyriopsis schlegeli, Pronodularia japanensis, Cristaria plicata, Cristaria tenuis, and Sinanodonta sp.) and two glochidia (Cristaria plicata and Cristaria tenuis) of unionid mussels. Jpn J Limnol 78: 87-96. (in Japanese with English summary)

Jang-Liaw NH, Tominaga K, Zhang C, Zhao Y, Nakajima J, Onikura N, Watanabe K (2019) Phylogeography of the Chinese false gudgeon, Abbottina rivularis, in East Asia, with special reference to the origin and artificial disturbance of Japanese populations. Ichthyol Res 66: 460-478.

Jolivet L (1992) Neogene kinematics in the Japan Sea region and the volcanic activity of the Northeast Japan arc. Proc Ocean Drill Program Sci Results 127: 1311-1331.

Kano Y, Tabata R, Nakajima J, Takada-Endo M, Zhang C, Zhao Y, Yamashita T, Watanabe K (2018) Genetic characteristics and possible introduced origin of the paradise fish Macropo- 
dus opercularis in the Ryukyu Archipelago, Japan. Ichthyol Res 65: 134-141.

Kano Y, Kurita Y, Kanno K, Saito K, Hayashi H, Onikura N, Yamasaki T (2019) Photo images, 3D/CT data and mtDNA of the freshwater mussels (Bivalvia: Unionidae) in the Kyushu and Ryukyu Islands, Japan, with SEM/EDS analysis of the shell. Biodiv Data J 7: e32114.

Kappes H, Haase P (2012) Slow, but steady: dispersal of freshwater molluscs. Aquat Sci 74: 1-14.

Kerswell AP (2006) Global biodiversity patterns of benthic marine algae. Ecology 87: 2479-2488.

Kitamura J (2005) Factors affecting seasonal mortality of rosy bitterling (Rhodeus ocellatus kurumeus) embryos on the gills of their host mussel. Popul Ecol 47: 41-51.

Kitamura J (2006a) Adaptive spatial utilization of host mussels by the Japanese rosy bitterling Rhodeus ocellatus kurumeus. J Fish Biol 69: 263-271.

Kitamura J (2006b) Reproductive ecology and host utilization of four sympatric bitterling (Acheilognathinae, Cyprinidae) in a lowland reach of the Harai River in Meiwa, Japan. Environ Biol Fish 78: 37-55.

Kondo T (2008) Monograph of Unionoida in Japan (Mollusca: Bivalvia). Malacological Society of Japan, Ibaraki, 69 pp.

Kondo T (2015) Catalogue of Japanese Unionid Shells in Takaki Kondo's Collection. Osaka Kyoiku University, Osaka, 58 pp. (in Japanese)

Krijgsman W, Hilgen FJ, Raffi I, Sierro FJ, Wilson DS (1999) Chronology, causes and progression of the Messinian salinity crisis. Nature 400: 652-655.

Kubota Y, Kusumoto B, Shiono T, Tanaka T (2017) Phylogenetic properties of Tertiary relict flora in the East Asian continental islands: imprint of climatic niche conservatism and in situ diversification. Ecography 40: 436-447.

Lanfear R, Frandsen PB, Wright AM, Senfeld T, Calcott B (2016) PartitionFinder 2: new methods for selecting partitioned models of evolution for molecular and morphological phylogenetic analyses. Mol Biol Evol 34: 772-773.

Lopes-Lima M, Froufe E, Do VT, Ghamizi M, Mock KE, Kebapçı Ü, Klishko O, Kovitvadhi S, Kovitvadhi U, Paulo OS, Pfeiffer JM, Raley M, Riccardi N, Şereflişan H, Sousa R, Teixeira A, Varandas S, Wu X, Zanatta DT, Zieritz A, Bogan AE (2017) Phylogeny of the most species-rich freshwater bivalve family (Bivalvia: Unionida: Unionidae): defining modern subfamilies and tribes. Mol Phylogenet Evol 106: 174-191.

Mills SC, Reynolds JD (2002a) Mussel ventilation rates as approximate cue for host selection by bitterling, Rhodeus sericeus. Oecologia 131: 473-478.

Mills SC, Reynolds JD (2002b) Host species preferences by bitterling, Rhodeus sericeus, spawning in freshwater mussels and consequences for offspring survival. Anim Behav 63: 10291036.

Mills SC, Taylor MI, Reynolds JD (2005) Benefits and costs to mussels from ejecting bitterling embryos: a test of the evolutionary equilibrium hypothesis. Anim Behav 70: 31-37.

Miyazaki JI, Dobashi M, Tamura T, Beppu S, Sakai T, Mihara M, Hosoya K (2011) Parallel evolution in eight-barbel loaches of the genus Lefua (Balitoridae, Cypriniformes) revealed by mitochondrial and nuclear DNA phylogenies. Mol Phylogenet
Evol 60: 416-427.

Motokawa M, Kajihara H (2016) Species Diversity of Animals in Japan. Springer Japan, Tokyo, 721 pp.

Nakajima T (1986) Pliocene cyprinid pharyngeal teeth from Japan and East Asia Neogene cyprinid zoogeography. In: Indo-Pacific Fish Biology, Proceedings of the Second International Conference on Indo-Pacific Fishes (eds Uyeno T, Arai R, Taniuchi T, Matsuura K), Ichthylogical Society of Japan, Ibaraki, pp. 502-513.

Nakajima T (1987) Freshwater fishes of Japan: their distribution, variation, and speciation. In: Formation and speciation of fish fauna in Lake Biwa (ed Nakajima T), Tokai University Press, Tokyo, pp. 215-229.

Nakano S, Kawabe T, Harayama S, Mizuno K, Takagi T, Komura R, Kumura K (2003) Geology of the Minakuchi district. Quadrangle Series, 1:50,000. Geological Survey of Japan, AIST, Tokyo, 83 pp. (in Japanese with English abstract)

Nguyen LT, Schmidt HA, von Haeseler A, Minh BQ (2014) IQTREE: a fast and effective stochastic algorithm for estimating maximum-likelihood phylogenies. Mol Biol Evol 32: 268-274.

Park JK, Kim W (2003) Two Corbicula (Corbiculidae: Bivalvia) mitochondrial lineages are widely distributed in Asian freshwater environment. Mol Phylogenet Evol 29: 529-539.

Pfeiffer JM, Graf DL (2013) Re-analysis confirms the polyphyly of Lamprotula Simpson, 1900 (Bivalvia: Unionidae). J Molluscan Stud 79: 249-256.

Rambaut A, Drummond AJ, Suchard M (2013) Tracer v1.6. Available at: http://tree.bio.ed. ac.uk/software/tracer/ (accessed on 17 February 2019)

Rees WJ (1965) The aerial dispersal of Mollusca. J Molluscan Stud 36: 269-282.

Reichard M, Ondračková M, Przybylski M, Liu H, Smith C (2006) The costs and benefits in an unusual symbiosis: experimental evidence that bitterling fish (Rhodeus sericeus) are parasites of unionid mussels in Europe. J Evol Biol 19: 788-796.

Reichard M, Przybylski M, Kaniewska P, Liu H, Smith C (2007) A possible evolutionary lag in the relationship between freshwater mussels and European bitterling. J Fish Biol 70: 709725.

Reichard M, Polačik M, Tarkan AS, Spence R, Gaygusuz Ö, Ercan E, Ondračková M, Smith C (2010) The bitterling-mussel coevolutionary relationship in areas of resent and ancient sympatry. Evolution 64: 3047-3056.

Ronquist F, Teslenko M, van der Mark P, Ayres DL, Darling A, Höhna S, Larget B, Liu L, Suchard MA, Huelsenbeck JP (2012) MrBayes 3.2: efficient Bayesian phylogenetic inference and model choice across a large model space. Syst Biol 61: 539-542.

Saito R, Jo J, Sekiné K, Bae YJ, Tojo K (2016) Phylogenetic analyses of the isonychiid mayflies (Ephemeroptera: Isonychiidae) in the northeast palearctic region. Entomol Res 46: 246-259.

Saito R, Kato S, Kuranishi RB, Nozaki T, Fujino T, Tojo K (2018) Phylogeographic analyses of the Stenopsyche caddisflies (Trichoptera: Stenopsychidae) of the Asian Region. Freshwater Sci 37: 562-572.

Saito T, Prozorova L, Sitnikova T, Surenkhorloo P, Hirano T, Morii Y, Chiba S (2018a) Molecular phylogeny of glacial relict species: a case of freshwater Valvatidae molluscs (Mol- 
lusca: Gastropoda) in North and East Asia. Hydrobiologia 818: $105-118$.

Saito T, Hirano T, Prozorova L, Do VT, Sulikowska-Drozd A, Sitnikova T, Surenkhorloo P, Yamazaki D, Morii Y, Kameda Y, Fukuda H, Chiba S (2018b) Phylogeography of freshwater planorbid snails reveals diversification patterns in Eurasian continental islands. BMC Evol Biol 18: 164.

Saito T, Do VT, Prozorova L, Hirano T, Fukuda H, Chiba S (2018c) Endangered freshwater limpets in Japan are actually alien invasive species. Conserv Genet 19: 947-958.

Sano I, Shirai A, Kondo T, Miyazaki JI (2017) Phylogenetic relationships of Japanese Unionoida (Mollusca: Bivalvia) based on mitochondrial 16S rDNA sequences. J Water Resour Prot 9: 493-509.

Satoguchi Y (2012) Geological History of Lake Biwa. In: Lake Biwa: Interactions between Nature and People (eds Kawanabe H, Nishino M, Maehata M), Springer, Berlin Heidelberg, pp. 9-16.

Shirai A, Kondo T, Kajita T (2010) Molecular markers reveal genetic contamination of endangered freshwater pearl mussels in pearl culture farms in Japan. Venus 68: 151-163.

Smith C, Reynolds JD Sutherland WJ (2000a) Population consequences of reproductive decisions. Proc R Soc Lond B 267: 1327-1334.

Smith C, Reynolds JD, Sutherland WJ, Jurajda P (2000b) Adaptive host choice and avoidance of superparasitism in the spawning decisions of bitterling (Rhodeus sericeus). Behav Ecol Sociobiol 48: 29-35.

Smith C, Rippon K, Douglas A, Jurajda P (2001) A proximate cue for oviposition site choice in the bitterling (Rhodeus sericeus). Freshw Biol 46: 903-911.

Strayer DL, Downing JA, Haag WR, King TL, Layzer JB, Newton TJ, Nichols JS (2004) Changing perspectives on pearly mussels, North America's most imperiled animals. BioScience 54: 429-439.

Suzuki T, Kitano T, Tojo K (2014) Contrasting genetic structure of closely related giant water bugs: phylogeography of Appasus japonicus and Appasus major (Insecta: Heteroptera, Belostomatidae). Mol Phylogenet Evol 72: 7-16.

Tamura K, Stecher G, Peterson D, Filipski A, Kumar S (2013) MEGA6: molecular evolutionary genetics analysis version 6.0. Mol Biol Evol 30: 2725-2729.

Tanabe AS (2008) MrBayes5D. Available at: https://www. fifthdimension.jp/products/mrbayes5d/ (Accessed on $22 \mathrm{De}$ cember 2018)

Taylor DW, Uyeno T (1965) Evolution of host specificity of freshwater salmonid fishes and mussels in the north pacific region. Venus 24: 199-209. (in Japanese with English summary)
Tojo K, Sekiné K, Takenaka M, Isaka Y, Komaki S, Suzuki T, Schoville SD (2017) Species diversity of insects in Japan: their origins and diversification processes. Entomol Sci 20: 357-381.

Tominaga K, Nakajima J, Watanabe K (2016) Cryptic divergence and phylogeography of the pike gudgeon Pseudogobio esocinus (Teleostei: Cyprinidae): a comprehensive case of freshwater phylogeography in Japan. Ichthyol Res 63: 79-93.

Torii M, Hayashida A, Otofuji Y (1985) The rotation of southwest Japan and the birth of the Sea of Japan. Kagaku 55: 4752. (in Japanese)

Tsao YF, Lin WW, Chang CH, Ueda T, Jang-Liaw NH, Zhao YH, Kao HW (2016) Phylogeography, historical demography, and genetic structure of the rose bitterling, Rhodeus ocellatus (Kner, 1866) (Cypriniformes: Acheilognathidae), in East Asia. Zool Stud 55: e49.

van Leeuwen CHA, van der Velde G, van Lith B, Klaassen M (2012) Experimental quantification of long distance dispersal potential of aquatic snails in the gut of migratory birds. PloS One 7: e32292.

van Leeuwen CHA, van der Velde G (2012) Prerequisites for flying snails: external transport potential of aquatic snails by waterbirds. Freshwater Sci 31: 963-972.

Walker KF, Jones HA, Klunzinger MW (2014) Bivalves in a bottleneck: taxonomy, phylogeography and conservation of freshwater mussels (Bivalvia: Unionoida) in Australasia. Hydrobiologia 735: 61-79.

Watanabe K, Uyeno T (1999) Fossil bagrid catfishes from Japan and their zoogeography, with description of a new species, Pseudobagrus ikiensis. Ichthyol Res 46: 397-412.

Watanabe K, Takahashi H, Kitamura A, Yokoyama R, Kitagawa T, Takeshima H, Sato S, Yamamoto S, Takehana Y, Mukai T, Ohara K, Iguchi K (2006) Biogeographical history of Japanese freshwater fishes: phylogeographic approaches and perspectives. Japan J Ichthyol 53: 1-38.

Wesselingh FP, Cadée GC, Renema W (1999) Flying high: on the airborne dispersal of aquatic organisms as illustrated by the distribution histories of the gastropod genera Tryonia and Planorbarius. Geol Mijnbouw 78: 165-174.

Whiting MF (2002) Mecoptera is paraphyletic: multiple genes and phylogeny of Mecoptera and Siphonaptera. Zool Scr 31: 93-104.

Yamada M, Ishibashi R, Kawamura K, Komaru A (2010) Interrelationships of the freshwater clams Corbicula leana Prime, 1864. and C. fluminea (Müller, 1774) distributed in Japan inferred from shell type and mitochondrial DNA Cyt $b$ region. Nippon Suisan Gakkaishi 76: 926-932. (in Japanese with English summary) 